The management of soft-tissue sarcomas requires particular skill by pathologists, surgeons, radiotherapists, and chemotherapists if optimal treatment is to be given. Ideally, all patients with these tumours should be treated in specialist oncology centres and whenever possible should be included in prospective randomised studies. While the evidence from the present series using historical controls is too preliminary to justify radical changes in managing these tumours, these results have warranted setting up prospective randomised trials. In time these should lead to standardisation of the treatment of patients presenting with localised disease. New drugs and combinations need continued testing in patients with advanced disease. These detailed studies of all stages of a rare tumour can be carried out effectively only by the co-operation of large centres in several countries.

1 Stout, A P, Annals of Surgery, 1946, 123, 447

2 Stout, A P, Cancer, 1948, 1, 30.

${ }^{3}$ Suit, H D, and Russell, W O, Cancer, 1975, 36, 759.

4 Pinedo, H M, and Kenis, Y, Cancer Treatment Reviews, 1977, 4, 67

5 Morton, D L, et al, Annals of Surgery, 1976, 184, 268.

${ }^{6}$ Suit, H D, Russell, W O, and Martin, R G, Cancer, 1975, 35, 1478

'Roseberg, S A, et al, Surgery, 1978, 84, 62.

${ }^{8}$ Karakousis, C P, et al, New York State Fournal of Medicine, 1979, 79, 328.

${ }^{9}$ Karakousis, C P, Holyoke, E D, and Mittelman, A, Proceedings of the American Association for Cancer Research, 1978, 19, 222.

${ }_{10}$ Didolkar, M S, et al, Annals of Surgery, 1978, 187, 332.

11 Gottlieb, J A, et al, Cancer Chemotherapy Reports, 1975, 6, 271.

\section{Medical treatment for hydatid disease?}

Until now the accepted treatment of hydatid disease has been some form of surgical drainage and removal of the cyst. Formalin or silver nitrate introduced into the cyst at operation to sterilise the contents before drainage and recently the cryogenic cone drainage technique ${ }^{1}$ have helped to reduce the risks of spillage of the cyst's contents, with secondary dissemination of the disease. Surgery may not always be possible, however, when the cysts are multiple or inaccessible-for example, in the liver-or when patients have already been subjected to many such operations. Furthermore, surgical intervention in malignant hydatid disease (caused by Echinococcus multilocularis), in which small cysts are disseminated throughout the liver, is rarely possible and has a high death rate.

The discovery of an effective oral or parenteral medication able to destroy the parasite and sterilise the cyst, for use either alone or in conjunction with surgery, has long been a cherished hope, and the introduction of mebendazole Vermox) may be a step towards this. This synthetic benzimiuazole derivative, related to the veterinary anthelmintics thiabendazole and cambendazole, is effective against several intestinal nematodes and cestodes, as well as the larvae of Trichinella spiralis ${ }^{2}$ and Taenia taeniaeformis. ${ }^{3}$ More recently the drug has been shown to be active against the larval stages of $E$ granulosus and $E$ multilocularis: it kills the parasite by limiting its glucose uptake and thus depleting its glycogen.

Experimentally, mebendazole has a lethal effect on the germinal membrane of the larvae in white mice infected with E granulosus, ${ }^{4}$ and it retards growth of intraperitoneal cysts as well as making them sterile. ${ }^{5}$ In vitro mebendazole diffuses passively through the cyst membrane of $E$ granulosus, ${ }^{6}$ and changes occur in the ultrastructure of germinal cells on electron microscopy after administration of the drug. ${ }^{7}$

The results of treating patients are encouraging. Bekhti and colleagues $^{8}$ gave mebendazole to four patients with hepatic hydatid disease in increasing doses, up to a maximum of 400-600 mg three times a day, during courses lasting 21-30 days. The intrahepatic cysts (monitored by ultrasound) completely regressed in four to 13 months, the patients improved clinically, and the concentrations of specific IgE against $E$ granulosus progressively fell. All the cysts were relatively small, however, none being over $5 \mathrm{~cm}$ in diameter. In clinical trials in Australia ${ }^{9} 37$ patients received mebendazole for hydatid disease, 35 of whom showed no clinical signs of recurrence or progression. Resolution of hepatic cysts on ultrasound computerised tomography scanning was observed in several cases, and at operation in another two patients the cysts appeared collapsed and contained non-viable parasites.

Not all reports, however, have been so encouraging, as was apparent at a recent workshop held by the Royal Society of Tropical Medicine and Hygiene. The largest clinical series was reported by French, who has treated 133 patients in Kenya with mebendazole before surgical drainage. Immediate postoperative complications, which included infection and formation of fistulas, affected only $7 \%$ of the treated patients compared with $14 \%$ of the 278 patients undergoing surgery alone; but the effectiveness of the drug in preventing recurrence of disease has still to be evaluated. What became clear during the workshop was that the natural history of hydatid disease is poorly documented. Furthermore, though the value of serial ultrasound and CT scanning for following changes in the size of cysts with treatment is not disputed, we do not know whether such changes indicate the death of the scolices they contain. In a small series of patients treated in Sardinia with mebendazole ( $30 \mathrm{mg} / \mathrm{kg}$ a day for four weeks), half the protoscolices were still alive on subsequent examination of the cyst contents at operation. Similarly, we need to assess the value of serology for monitoring the effectiveness of this drug. Proof that the parasites have lost their viability from direct histological examination of the cyst's contents is at present the only criterion we have against which new tests can be assessed.

So far, mebendazole has been well tolerated in the high doses used, with only occasional reports of gastric irritation, pruritus, rash (including exfoliative dermatitis), and transient abnormalities found in liver function tests. Respiratory symptoms with cough and fever were reported in eight of the 133 Kenyan patients, and histologically confirmed glomerulonephritis in six-possibly as a result of immune complexes liberated into the circulation. Trials carried out on an international basis, as discussed at the workshop, should help to answer some of the many questions on the use of this drug in human hydatid disease.

${ }^{1}$ Saidi, F, Annals of the Royal College of Surgeons of England, 1977, 59, 115.

2 Thienpont, D, et al, in Trichinellosis, ed Ch W Kim. New York, State University Press, 1974.

- Thienpont, D, Vanparijs, O, and Hermans, L, fournal for Parasitology, $1974,60,1052$.

- Heath, D D, Christie, M J, and Chevis, R A, Parasitology, 1975, 70, 273.

- Vanparijs, O, Biological Research Report No 11087. High Wycombe, Janssen Pharmaceutica, 1976.

- Reisin, I L, et al, International fournal for Parasitology, 1977, 7, 189.

${ }^{7}$ Kammerer, W S, and Judge, D M, American fournal of Tropical Medicine and Hygiene, 1976, 25, 714.

${ }^{8}$ Bekhti, A, et al, British Medical fournal, 1977, 2, 1047.

- Beard, T C, Rickard, M D, and Goodman, H T, Medical fournal of Australia , 1978, 1, 633. 